\title{
Performance Limitations Analysis of Imperfect Attenuators for Adaptive Self-Interference Cancellation System
}

\author{
Jiancheng LIU, Houde QUAN, Peizhang CUI, Huixian SUN \\ Dept. of Information Engineering, Mechanical Engineering College, Heping West Road No.97, 050003 Shijiazhuang, China \\ liujiancheng1987@sina.com.cn \\ Manuscript received March 11, 2016
}

\begin{abstract}
Radio frequency $(R F)$ adaptive self-interference cancellation system (RFAICS) is extensively used to suppress the self-interference of radios operating in the same platform, such as military command vehicles, airplanes and navy vessels. RFAICS is generally consisted of couplers, attenuators, delay units, amplifiers, and filters and so on. However, RFAICS usually suffers severely from the imperfect attenuators. This paper firstly explores the RFAICS operation process in theory, and then thoroughly investigates and analyzes the negative effects of non-ideal attenuators on performance of RFAICS. The closed-form expressions fully describing the influences of attenuation bias and response-time respectively on the interference cancellation ratio (ICR) and system convergence time (SCT) are developed with this aim. Simulations are provided for the validity of the limitation analysis and $o b$ tained expressions. Results of simulations are in agreement with theoretical analysis, which is significant for component configuration in taking RFAICS into practice.
\end{abstract}

\section{Keywords}

Self-interference, adaptive interference cancellation, interference cancellation ratio, system convergence time

\section{Introduction}

Tactical vehicle and navy shipboard systems commonly consist of several different radios, which are demanded to transmit and receive signals simultaneously. Although the frequencies of these radios are separated, the transmitters would jam receivers because their transmitting signals are much stronger than the desired signal from remote transmitters. The interference between radios on the same platform is also called as electromagnetic interference (EMI) [1], and this interference generated from neighbor radios is the major roadblock to cooperation and compatibility of radios in specified nearby frequencies [1], [2]. Therefore, efficient self-interference cancellation is significant for the radios suc- cessfully and simultaneously operating in the same platform and for remarkable improvement in spectrum efficiency.

In the last decades, researchers have made great contributions to the adaptive interference cancellation system (AICS) [2-10], and these studies are focused on not only military application but also civil communication systems. [2] employs a RF adaptive self-interference cancellation system (RFAICS) based on controlling and mixing two orthogonal signals derived from transmitted signal, and improves the power handling capability of signal controller. The broadband cancellation technology for adaptive UHF (225$400 \mathrm{MHz}$ ) interference cancellation systems is proposed in [3], and the cancellation system can be employed with tunable or hopping transmitters. The self-interference of communication sites containing frequency hopping (FH) and single channel radios is analyzed in [4]. Furthermore, AICS is also applied in electrocardiography to cancel interference [5], and RFAICS is also the critical self-interference suppression method for civil co-time and co-frequency full-duplex communication systems [6-8]. On the whole, the adaptive cancellation methods of above literatures are all based on minimum mean square error (MMSE) criterion. Another interference cancellation system is proposed in [9], [10]. In this distinctive scheme, an additional transmit chain is built to send a canceling signal. The canceling signal is controlled in baseband to add with self-interference signal at the receive antenna, yet the achieved ICR is low due to inevitable distortion between the two RF transmit chains. Another new cancellation circuit and tuning algorithm proposed in [11] could obtain about $45 \mathrm{~dB}$ ICR, while this circuit is more complicated and its delays are hard to adjust exactly. In addition, [12-14] investigate and analyze the performance of RFAICS in different ways. [12] establishes RFAICS model in time-domain for analyzing the stability, interference cancellation ratio (ICR), desired signal distortion and system convergence time (SCT). The performance of RFAICS is also studied in [13] in terms of ICR, SCT and desired signal cancellation ratio (SCR). It is found that the RFAICS feedback circuit should be in the condition of low damping in order to reduce the negative effect of desired signals. In [14], a novel convex reformulation for 
tuning the attenuators is presented, however, the attenuators and couplers are assumed as ideal devices.

To the best of the authors' knowledge, the negative effects of imperfect amplitude attenuators and delay units have not been completely studied for RFSICS, while these analog components are key factors for implementing RFAICS in practice, especially for attenuators. In this paper, the theoretical analysis of imperfect attenuator impacts on RFAICS is completely presented. The main contributions of this paper can be summarized as follows. Firstly, we mathematically derive the convergence process and stability of RFAICS based on the typical scheme, and the ICR expression is achieved. Furthermore, how attenuator accuracy influences ICR of RFAICS and attenuator response-time determines SCT of adaptive cancellation are investigated and analyzed. Consequently, the closed expressions of ICR and SCT with respect to attenuation bias and attenuator response-time respectively are achieved. Finally, simulations are performed to demonstrate the validity of proposed ICR and SCT expressions, which have great significance for selecting appropriate attenuators according to demands of different RFAICSs.

The rest of this paper is organized as follows. Section 2 describes the structure and operating principle of typical RFAICS. In Sec. 3, theoretical analysis of RFAICS convergence process is exhaustively shown to obtain ICR and SCT expressions, and then RFAICS performance degeneration expressions caused by imperfect attenuators are derived based on the ICR and SCT expressions. In Sec. 4, simulations for various scenarios are provided. The results demonstrate the validity of derived expressions. Section 5 concludes the work of this paper and indicates further studies.

\section{RF Adaptive Interference Cancella- tion System}

To ensure simultaneous and successful operation of radios in the same platform, which have limited frequency separation bands, the adaptive self-interference cancellation is necessary and should be completed in RF domain before frequency down conversion to avoid blocking receive channel. Generally, the adaptive interference cancellation system is consisted of a sample coupler, delay unit, phase shifter, two attenuators, delay estimation devices, attenuation values calculation devices and cancellation coupler [2], [3], [7], which is shown in Fig. 1.

In this adaptive cancellation system, it is assumed that $h(t)$ is the channel response of self-interference and mainly consists of the direct propagation path. $s_{\mathrm{RF}}(t)$ is the reference signal extracted from sample coupler in front of transmit antenna. $r(t)$ is the received signal, which contains self-interference $s_{\mathrm{I}}(t)$, desired signal $d(t)$ and additive white Gaussian noise $n(t)$. It is noticed that $s_{\mathrm{I}}(t)$ and $d(t)$ occupy different frequency bands.

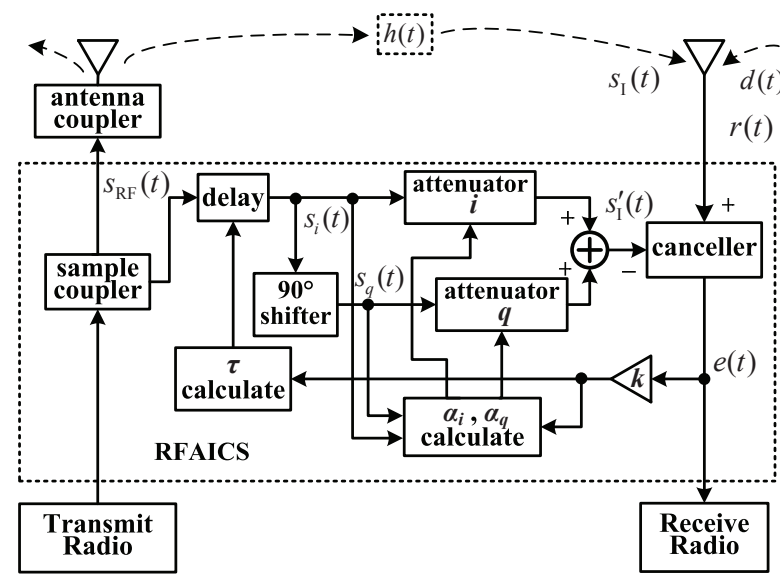

Fig. 1. Principal illustration of the typical RF adptive interference cancellation system, mainly consisted of attenuators, time delay unit and couplers.

The self-interference $s_{\mathrm{I}}(t)$ and received signal $r(t)$ can be written respectively as [15]

$$
s_{\mathrm{I}}(t)=s_{\mathrm{RF}}(t) * h(t)=\int_{0}^{+\infty} s_{\mathrm{RF}}(t-\tau) h(\tau) d \tau
$$

and

$$
\begin{aligned}
r(t) & =s_{\mathrm{I}}(t)+d(t)+n(t) \\
& =\int_{0}^{+\infty} s_{\mathrm{RF}}(t-\tau) h(\tau) d \tau+d(t)+n(t) .
\end{aligned}
$$

Obviously, the goal of RFAICS is to subtract $s_{\mathrm{I}}(t)$ from $r(t)$ through perfectly estimating self-interference channel $h(t)$. Considering antennas of transmit and receive radios are fixed and close, $h(t)$ would mainly change the amplitude attenuation and propagation delay of interference $s_{\mathrm{I}}(t)$. It is noticed that the delay unit is commonly constant since the transmit and receive antennas are fixed. Consequently, adaptive interference cancellation should aim to mainly exploit attenuators to reconstruct canceling signal $s_{\mathrm{I}}^{\prime}(t)$.

The reference signal $s_{\mathrm{RF}}(t)$ passes through a fixed delay $\tau$, and then it is divided into two orthogonal signals $s_{i}(t)$ and $s_{q}(t)$. One of them remains unchanged to the reference signal, and the other is converted by a $90^{\circ}$ phase shift. The two signals are respectively controlled by two attenuators $\left(\alpha_{i}\right.$ and $\alpha_{q}$ ) to synthesize the canceling signal $s_{\mathrm{I}}^{\prime}(t)$, which will be added with the received signal from the receive antenna. Furthermore, the output signal of the canceller is called error signal consisting of desired signal, residual interference and noise. This error signal is utilized to adjust the two attenuators. It is assumed that $\alpha_{i}(t)$ and $\alpha_{q}(t)$ are respectively the attenuation coefficients of the two attenuators at the time $t$, and $e(t)$ is the output error signal of canceller. The RFAICS operation process can be briefly expressed as

$$
\begin{aligned}
e(t) & =r(t)-s_{\mathrm{I}}^{\prime}(t) \\
& =s_{\mathrm{I}}(t)-s^{\prime}{ }_{\mathrm{I}}(t)+d(t)+n(t), \\
s_{\mathrm{I}}^{\prime}(t) & =\left[\alpha_{i}(t) \alpha_{q}(t)\right] \cdot\left[\begin{array}{c}
s_{i}(t) \\
s_{q}(t)
\end{array}\right],
\end{aligned}
$$




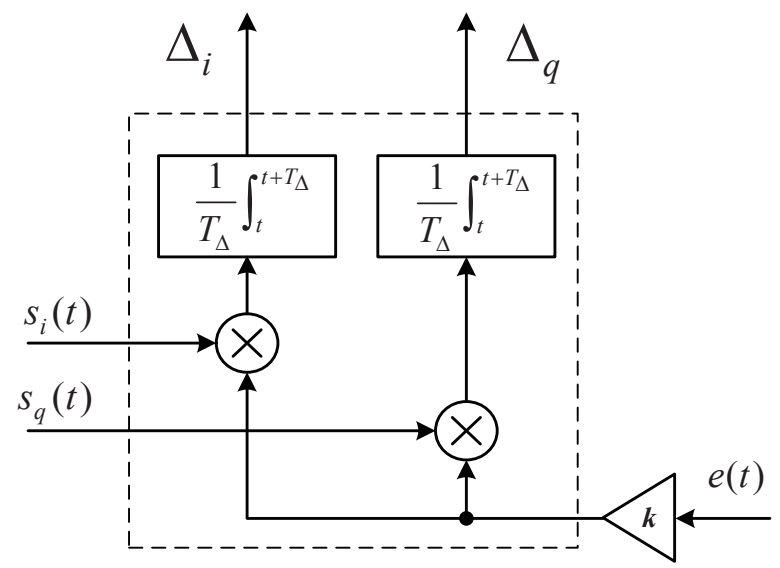

Fig. 2. Principal diagram of attenuation increments calculation, mainly depending on the reference signals and residual signal of cancellation.

$$
\left[\begin{array}{c}
\alpha_{i}\left(t+T_{\Delta}\right) \\
\alpha_{q}\left(t+T_{\Delta}\right)
\end{array}\right]=\left[\begin{array}{c}
\alpha_{i}(t) \\
\alpha_{q}(t)
\end{array}\right]+\left[\begin{array}{c}
\Delta_{i}(t) \\
\Delta_{q}(t)
\end{array}\right]
$$

where $\Delta_{i}(t)$ and $\Delta_{q}(t)$ are respectively the increments of $\alpha_{i}(t)$ and $\alpha_{q}(t), T_{\Delta}$ is the execution interval time consumed by adjusting attenuators once completely, which is related with the attenuator response-time and duration of calculating $\Delta_{i}(t)$ and $\Delta_{q}(t)$. The calculation of $\Delta_{i}(t)$ and $\Delta_{q}(t)$ is given in (6) and illustrated in Fig. 2.

$$
\left[\begin{array}{c}
\Delta_{i}(t) \\
\Delta_{q}(t)
\end{array}\right]=\frac{k}{T_{\Delta}} \int_{t}^{t+T_{\Delta}}\left[e(t) s_{i}(t) e(t) s_{q}(t)\right]^{\mathrm{T}} \mathrm{d} t
$$

where $\mathrm{T}$ denotes the transpose of vector or matrix, and the integration time equals to the interval time $T_{\Delta}$ in (5), $k$ is the adjustment factor of cancellation loop, and $T_{\Delta}$ should be kept short as long as the stability of RFAICS is not deteriorated.

It should be noted that the above mathematical description of adaptive cancellation is equivalent to the least mean square (LMS) process, based on the MMSE criterion,

$$
\min \left\{\mathrm{E}\left(|e(t)|^{2}\right)\right\}=\min \left\{\mathrm{E}\left(\left|r(t)-s_{\mathrm{I}}^{\prime}(t)\right|^{2}\right)\right\}
$$

where $\mathrm{E}(x)$ denotes the statistical mean value of $x$, and $|x|^{2}$ denotes the absolute value square of $x$.

\section{RFAICS Performance Analysis for Imperfect Key Analog Components}

In this section, we first establish models for analyzing the convergence process and stability of RFAICS based on (3)-(6) and derive the final ICR expression. Based on the analysis results, two critical indicators for evaluating RFAICS are demonstrated: ICR degeneration caused by attenuation bias and effect of attenuator response-time on SCT.

\subsection{Convergence and Stability Analysis of RFAICS}

To understand adaptive interference cancellation completely, the studies of its convergence process and stability are indispensable, including the derivation of ICR and SCT for RFAICS. Now we theoretically describe RFAICS convergence and exploit the model in Fig. 1 as the research fundament.

It is common that the self-interference and desired signal usually have the different radio frequencies, but could have the same data rate, the same coding types and the same modulation schemes. Without the loss of generality, we assume that the self-interference and the desired signal adopt QPSK modulation. From Fig. 1 it can be observed that signals $s_{i}(t)$ and $s_{q}(t)$ are orthogonal reference signals, and are both extracted from the transmitting signal $s_{\mathrm{RF}}(t)$. Moreover, $s_{i}(t)$ and $s_{q}(t)$ are random cyclostationary signals, and their statistics mean value and power are respectively assumed as zero and $P_{\mathrm{s}}$. Their expressions are written as

$$
\left\{\begin{array}{l}
s_{i}(t)=\sum_{m}\left[g(t-m T) \cos \left(\omega t+\theta_{m}\right)\right] \\
s_{q}(t)=\sum_{m}\left[g(t-m T) \sin \left(\omega t+\theta_{m}\right)\right]
\end{array}\right.
$$

where $\theta_{m}$ is modulated phase of the $m$ th baseband symbol with period $T, g(t)$ is the pulse shape of baseband symbol. For the sake of simplicity, the pulse shape is assumed rectangular pulse with period $T$,

$$
g(t)= \begin{cases}1 & -\frac{T}{2} \leq t<\frac{T}{2}, \\ 0 & \text { others. }\end{cases}
$$

We first deduce the convergence process with the assumption that the time delay bias is zero, i.e., the estimation of self-interference propagation delay $\tau$ is accurate. Hence, the self-interference involved in the received signal $r(t)$ can be expressed as

$$
s_{\mathrm{I}}(t)=\left[\begin{array}{ll}
\beta_{i} & \beta_{q}
\end{array}\right] \cdot\left[\begin{array}{ll}
s_{i}(t) & s_{q}(t)
\end{array}\right]^{\mathrm{T}}=\boldsymbol{\beta}^{\mathrm{T}} \boldsymbol{s}(t)
$$

where $s(t)=\left[\begin{array}{ll}s_{i}(t) & s_{q}(t)\end{array}\right]^{\mathrm{T}}$ is orthogonal signal vector, $\boldsymbol{\beta}=\left[\begin{array}{ll}\beta_{i} & \beta_{q}\end{array}\right]^{\mathrm{T}}$ denotes the amplitude attenuation vector compared to the reference signal vector $s(t)$. The received self-interference power is calculated as $P_{\mathrm{I}}=\left(\beta_{i}^{2}+\beta_{q}^{2}\right) P_{\mathrm{s}}$, and $\beta$ depends on the self-interference propagation channel and the power of transmitted signal.

We define $\boldsymbol{\alpha}(t)=\left[\begin{array}{ll}\alpha_{i}(t) & \alpha_{q}(t)\end{array}\right]^{\mathrm{T}}$ and $\boldsymbol{m}(t)=\boldsymbol{\beta}-\boldsymbol{\alpha}(t)$. Considering the irrelevant property between $\boldsymbol{m}(t), n(t), d(t)$ and $s(t)$, and the statistics mean values of $n(t)$ and $d(t)$ are zeros [16], the statistics mean-square value of error signal $e(t)$ can be given by

$$
\begin{aligned}
\mathrm{E}\left\{|e(t)|^{2}\right\}= & \mathrm{E}\left\{\begin{array}{l}
\boldsymbol{m}^{\mathrm{T}}(t) \boldsymbol{s}(t) \boldsymbol{s}^{\mathrm{T}}(t) \boldsymbol{m}(t)+ \\
2 \boldsymbol{m}^{\mathrm{T}}(t) \boldsymbol{s}(t)[d(t)+n(t)]+ \\
{[d(t)+n(t)]^{2}}
\end{array}\right\} \\
= & \mathrm{E}\left\{\boldsymbol{m}^{\mathrm{T}}(t) \cdot \mathrm{E}\left[\boldsymbol{s}(t) \boldsymbol{s}^{\mathrm{T}}(t)\right] \cdot \boldsymbol{m}(t)\right\}+ \\
& \mathrm{E}\left\{[d(t)+n(t)]^{2}\right\} .
\end{aligned}
$$


By denoting

$$
\boldsymbol{R}=\mathrm{E}\left[\boldsymbol{s}(t)^{\mathrm{T}} \boldsymbol{s}(t)\right]=\left(\begin{array}{cc}
P_{\mathrm{s}} & 0 \\
0 & P_{\mathrm{s}}
\end{array}\right) .
$$

The expression (11) can be further written as

$$
\mathrm{E}\left\{|e(t)|^{2}\right\}=\mathrm{E}\left\{\boldsymbol{m}^{\mathrm{T}}(t) \cdot \boldsymbol{R} \cdot \boldsymbol{m}(t)\right\}+\left(P_{\mathrm{d}}+\sigma_{\mathrm{n}}^{2}\right)
$$

where $P_{\mathrm{d}}$ and $\sigma_{\mathrm{n}}^{2}$ respectively denote the desired signal $d(t)$ power and additive white noise $n(t)$ power.

Since the integration in (6) is equivalent to mean value calculation, we can ignore it for analyzing (13). Thus we simplify expression (5) as

$$
\alpha_{\Delta}(t)=\left[\begin{array}{c}
\Delta_{i}(t) \\
\Delta_{q}(t)
\end{array}\right] \approx k \cdot e(t) s(t)
$$

Combining (5) and (6), it is obtained as

$$
\begin{aligned}
\boldsymbol{m}\left(t+T_{\Delta}\right) & =\boldsymbol{m}(t)-\boldsymbol{\alpha}_{\Delta}(t) \\
& =\boldsymbol{m}(t)-k\left[\boldsymbol{m}^{\mathrm{T}}(t) \boldsymbol{s}(t)+d(t)+n(t)\right] \cdot \boldsymbol{s}(t) \\
& =\left[\boldsymbol{I}-k \boldsymbol{s}(t) \boldsymbol{s}^{\mathrm{T}}(t)\right] \boldsymbol{m}(t)-k \boldsymbol{s}(t) \cdot[d(t)+n(t)] .
\end{aligned}
$$

Given (15), (13) can be expressed by $\boldsymbol{m}\left(t-T_{\Delta}\right)$ as

$$
\begin{aligned}
& \mathrm{E}\left\{|e(t)|^{2}\right\}=\mathrm{E}\left\{\begin{array}{l}
\boldsymbol{m}^{\mathrm{T}}\left(t-T_{\Delta}\right) \cdot\left[\boldsymbol{I}-k \boldsymbol{s}(t) \boldsymbol{s}^{\mathrm{T}}(t)\right]^{\mathrm{T}} \cdot \\
\boldsymbol{R} \cdot\left[\boldsymbol{I}-k \boldsymbol{s}(t) \boldsymbol{s}^{\mathrm{T}}(t)\right] \boldsymbol{m}\left(t-T_{\Delta}\right)
\end{array}\right\}+ \\
& \mathrm{E}\left\{\begin{array}{l}
k^{2} \boldsymbol{s}^{\mathrm{T}}(t) \cdot \boldsymbol{R} \cdot \\
\boldsymbol{s}(t)[d(t)+n(t)]^{2}
\end{array}\right\}+P_{\mathrm{d}}+\sigma_{\mathrm{n}}^{2} \\
& =\mathrm{E}\left\{\begin{array}{l}
\boldsymbol{m}^{\mathrm{T}}\left(t-T_{\Delta}\right) \cdot \mathrm{E}\left\{\left[\boldsymbol{I}-k \boldsymbol{s}(t) \boldsymbol{s}^{\mathrm{T}}(t)\right]^{2}\right\} \\
\boldsymbol{R} \cdot \boldsymbol{m}\left(t-T_{\Delta}\right)
\end{array}\right\}+ \\
& 2 k^{2} P_{\mathrm{s}}^{2}\left(P_{\mathrm{d}}+\sigma_{\mathrm{n}}^{2}\right)+P_{\mathrm{d}}+\sigma_{\mathrm{n}}^{2} \\
& =\mathrm{E}\left\{\boldsymbol{m}^{\mathrm{T}}\left(t-T_{\Delta}\right) \cdot \boldsymbol{R} \cdot[\boldsymbol{I}-k \boldsymbol{R}]^{2} \cdot \boldsymbol{m}\left(t-T_{\Delta}\right)\right\}+ \\
& 2 k^{2} P_{\mathrm{s}}^{2}\left(P_{\mathrm{d}}+\sigma_{\mathrm{n}}^{2}\right)+P_{\mathrm{d}}+\sigma_{\mathrm{n}}^{2} \text {. }
\end{aligned}
$$

It is assumed that the convergence process has continued time $t=n T_{\Delta}$, meanwhile $\mathrm{E}\left\{|e(t)|^{2}\right\}$ has gone through $n$ iterations, and (16) could be expressed as

$$
\begin{aligned}
\mathrm{E}\left\{|e(t)|^{2}\right\}= & \\
\left(1-k P_{\mathrm{s}}\right)^{2 n} \cdot\left\{\mathrm{E}\left(\left|e\left(t_{0}\right)\right|^{2}\right)-\right. & \left.\frac{\left(2+k P_{\mathrm{s}}\right)\left(P_{\mathrm{d}}+\sigma_{\mathrm{n}}^{2}\right)}{2-k P_{\mathrm{s}}}\right\} \\
+ & \frac{\left(2+k P_{\mathrm{s}}\right)\left(P_{\mathrm{d}}+\sigma_{\mathrm{n}}^{2}\right)}{2-k P_{\mathrm{s}}}
\end{aligned}
$$

where, $\mathrm{E}\left\{\left|e\left(t_{0}\right)\right|^{2}\right\}$ denotes the statistics mean-square value of initial error signal. Obviously, the values of reference signal power $P_{\mathrm{s}}$, fixed desired signal power $P_{\mathrm{d}}$, noise power $\sigma_{\mathrm{n}}^{2}$ and specific $\mathrm{E}\left\{\left|e\left(t_{0}\right)\right|^{2}\right\}$ are all constant. As a result, (17) is absolutely determined by adjustment factor $k$. If it is assumed that the initial values of the two attenuators are both zero, statistics mean-square value of initial error signal $\mathrm{E}\left\{\left|e\left(t_{0}\right)\right|^{2}\right\}$ is equivalent to the power of received signal, and written as

$$
\begin{aligned}
\mathrm{E}\left\{\left|e\left(t_{0}\right)\right|^{2}\right\} & =\mathrm{E}\left\{|r(t)|^{2}\right\} \\
& =\left(\beta_{i}^{2}+\beta_{q}^{2}\right) P_{\mathrm{s}}+P_{\mathrm{d}}+\sigma_{\mathrm{n}}^{2} .
\end{aligned}
$$

We define ICR as the ratio of the received selfinterference power and the residual self-interference power, and the residual self-interference is the error signal except the desired signal and noise. Based on (17) and (18), the ICR value at $t=n T_{\Delta}$ can be calculated as

$$
\begin{aligned}
\mathrm{ICR} & =10 \log _{10}\left(\frac{\mathrm{E}\left(\left|e\left(t_{0}\right)\right|^{2}\right)-P_{\mathrm{d}}-\sigma_{\mathrm{n}}^{2}}{\mathrm{E}\left(|e(t)|^{2}\right)-P_{\mathrm{d}}-\sigma_{\mathrm{n}}^{2}}\right) \\
& =10 \log _{10}\left(\frac{P_{\mathrm{I}}}{\mathrm{E}\left(|e(t)|^{2}\right)-P_{\mathrm{d}}-\sigma_{\mathrm{n}}^{2}}\right) .
\end{aligned}
$$

In what follows, we use (19) to analyze effects of attenuator bias and response-time. According to the analysis of LMS adjustment factor $k$ in [16], the power of final error signal $\mathrm{E}\left\{|e(t)|^{2}\right\}$ can converge to a stable value as long as $k$ is rational. Given (17), it is found that if $\left|1-k P_{\mathrm{s}}\right|<1$, we have $\left(1-k P_{\mathrm{s}}\right)^{2 n} \rightarrow 0$. Thus when $n$ is large enough, in other words, the cancellation system has arrived at its stability state, the statistics mean-square value is obtained by $\mathrm{E}\left\{|e(t)|^{2}\right\} \approx\left(2+k P_{\mathrm{s}}\right)\left(P_{\mathrm{d}}+\sigma_{\mathrm{n}}^{2}\right) /\left(2-k P_{\mathrm{s}}\right)$, and the ICR can be simplified as

$$
\mathrm{ICR}_{\infty}=10 \log _{10}\left(\frac{\left(\beta_{i}^{2}+\beta_{q}^{2}\right)\left(2-k P_{\mathrm{s}}\right)}{2 k\left(P_{\mathrm{d}}+\sigma_{\mathrm{n}}^{2}\right)}\right) .
$$

It is clear that the ICR of RFAICS stability increases as adjustment factor $k$ decreases, however the SCT would also increase as adjustment factor $k$ decreases, which is analyzed in subsection 3.3, and this effect is unexpected.

\subsection{Negative Effect of Attenuator Bias on ICR}

Considering the characteristics of practical attenuators [17], [18], the attenuator bias model is complicated, and the attenuation error is affected by many factors. Generally, we focus on the variance and mean of attenuation error in most applications, and we assume the biases of two attenuators, refering to [17], are independent and obey the normal distribution with the mean zero and variance $\sigma_{\varepsilon}^{2}$. The vector form of two attenuator biases is $\varepsilon(t)=\left[\begin{array}{cc}\varepsilon_{i}(t) & \varepsilon_{q}(t)\end{array}\right]^{\mathrm{T}}$. Taking into account attenuation bias, we define the error signal as $e_{\mathrm{a}}(t)$. Based on the above assumption, (14) with attenuator biases can be rewritten as

$$
\boldsymbol{\alpha}^{\prime}{ }_{\Delta}(t)=k e_{\mathrm{a}}(t) \boldsymbol{s}(t)+\boldsymbol{\varepsilon}(t) .
$$


Therefore, (15) should be rewritten as

$$
\begin{aligned}
\boldsymbol{m}^{\prime}\left(t+T_{\Delta}\right)= & {\left[\boldsymbol{I}-k \boldsymbol{s}(t) \boldsymbol{s}^{\mathrm{T}}(t)\right] \cdot \boldsymbol{m}^{\prime}(t)-} \\
& k \boldsymbol{s}(t)[d(t)+n(t)]-\boldsymbol{\varepsilon}(t) .
\end{aligned}
$$

It is similar to (14) that the statistics mean-square value of error signal affected by attenuator bias $\mathrm{E}\left\{\left|e_{\mathrm{a}}(t)\right|^{2}\right\}$ can be expressed as (23), since the attenuator bias could be supposed as uncorrelated with $n(t), d(t), \boldsymbol{s}(t)$ and $\boldsymbol{m}^{\prime}(t)$.

$$
\begin{aligned}
\mathrm{E}\left\{\left|e_{\mathrm{a}}(t)\right|^{2}\right\}= & \mathrm{E}\left\{\boldsymbol{m}^{\prime \mathrm{T}}\left(t-T_{\Delta}\right) \boldsymbol{R}[\boldsymbol{I}-k \boldsymbol{R}]^{2} \boldsymbol{m}^{\prime}\left(t-T_{\Delta}\right)\right\}+ \\
& 2 k^{2} P_{\mathrm{s}}^{2}\left(P_{\mathrm{d}}+\sigma_{\mathrm{n}}^{2}\right)+2 P_{\mathrm{s}} \sigma_{\varepsilon}^{2}+P_{\mathrm{d}}+\sigma_{\mathrm{n}}^{2} .
\end{aligned}
$$

Further, $\mathrm{E}\left\{\left|e_{\mathrm{a}}(t)\right|^{2}\right\}$ with $t=n T_{\Delta}$ is written as

$$
\begin{aligned}
\mathrm{E}\left\{\left|e_{\mathrm{a}}(t)\right|^{2}\right\} & =\left(1-k P_{\mathrm{s}}\right)^{2 n} \cdot \mathrm{E}\left(\left|e\left(t_{0}\right)\right|^{2}\right)- \\
& \left(1-k P_{\mathrm{s}}\right)^{2 n} \cdot \frac{2 \sigma_{\varepsilon}^{2}+k\left(P_{\mathrm{d}}+\sigma_{\mathrm{n}}^{2}\right)\left(2+k P_{\mathrm{s}}\right)}{k\left(2-k P_{\mathrm{s}}\right)}+ \\
& \frac{2 \sigma_{\varepsilon}^{2}+k\left(P_{\mathrm{d}}+\sigma_{\mathrm{n}}^{2}\right)\left(2+k P_{\mathrm{s}}\right)}{k\left(2-k P_{\mathrm{s}}\right)} .
\end{aligned}
$$

Consequently, the asymptotic and steady ICRs with attenuation bias are obtained respectively

$$
\begin{aligned}
\mathrm{ICR}^{\prime} & =10 \log _{10}\left(\frac{P_{\mathrm{I}}}{\mathrm{E}\left(\left|e_{\mathrm{a}}(t)\right|^{2}\right)-P_{\mathrm{d}}-\sigma_{\mathrm{n}}^{2}}\right) \\
& =10 \log _{10}\left(\frac{\left(\beta_{i}^{2}+\beta_{q}^{2}\right) P_{\mathrm{s}}}{\mathrm{E}\left(\left|e_{\mathrm{a}}(t)\right|^{2}\right)-P_{\mathrm{d}}-\sigma_{\mathrm{n}}^{2}}\right), \\
\mathrm{ICR}_{\infty}^{\prime} & =10 \log _{10}\left(\frac{\left(\beta_{i}^{2}+\beta_{q}^{2}\right) k P_{\mathrm{s}}\left(2-k P_{\mathrm{s}}\right)}{2 \sigma_{\varepsilon}^{2}+2 k^{2} P_{\mathrm{s}}\left(P_{\mathrm{d}}+\sigma_{\mathrm{n}}^{2}\right)}\right)
\end{aligned}
$$

where calculations of (25) and (26) are based on (18) and (24). Obviously, the stability ICR with attenuation bias degenerates as the variance of attenuation bias increases. The ICR would be more seriously affected by attenuation bias if the adjustment factor reduces. Moreover, the stability ICR can be estimated through the prior knowledge of attenuation bias, adjustment factor, reference signal power, self-interference power, desired signal power and noise variance.

\subsection{Impact of Attenuator Response-Time on SCT}

For some applications, such as frequency hopping and burst communications, SCT is a critical property of RFAICS, which is subject to attenuation adjustment time, i.e. the attenuator response-time. However, to our best knowledge, SCT of RFAICS has not been studied. Hence, it is necessary to analyze the impact of attenuator response-time on SCT. Attenuation bias is not taken into account in this subsection for simplicity.
If we assume $T_{\mathrm{s}}$ is the response-time of attenuator, the interval time $T_{\Delta}$ in (5) to completely adjust attenuators once should satisfy $T_{\Delta}>T_{\mathrm{s}}$. Therefore, the interval time can take the place of attenuator response-time in analysis. Generally, we determine whether the cancellation operation has been the stability by ICR value of expression (19). When ICR is larger than the assumed threshold $\gamma$, the state of RFAICS is regarded as stability; otherwise the RFAICS is at the convergence state. The SCT $t_{\mathrm{c}}$ of RFAICS can be obtained by combining (17), (18) and (19) as

$t_{\mathrm{c}}=T_{\Delta} \log _{v}\left\{\frac{\left(2-k P_{\mathrm{s}}\right)\left(\beta_{i}^{2}+\beta_{q}^{2}\right) \cdot 10^{-\frac{\gamma}{10}}-2 k\left(P_{\mathrm{d}}+\sigma_{\mathrm{n}}^{2}\right)}{\left(2-k P_{\mathrm{s}}\right)\left(\beta_{i}^{2}+\beta_{q}^{2}\right)-2 k\left(P_{\mathrm{d}}+\sigma_{\mathrm{n}}^{2}\right)}\right\}$

where $v=\left(1-k P_{\mathrm{s}}\right)^{2}$.

It is well known that if the adjustment factor $k$ is fixed, the value of $t_{\mathrm{c}}$ is mainly influenced by $T_{\Delta}$. In other words, $t_{\mathrm{c}}$ is determined by attenuator response-time $T_{\mathrm{S}}$.

At this point, all negative effects of attenuation bias and attenuator response-time are formulated and analyzed in detail, and the derived expressions directly reflect the relationships between them and ICR, SCT. Furthermore, the achieved results in this section can also be applied on analyzing the popular full-duplex communication system, for which the RF adaptive self-interference cancellation is indispensable. The transmitting and receiving carrier frequencies are only the difference between full-duplex and the tactical wireless radio communication, and the transmitted and received signals in full-duplex system are also irrelevant [9], [11]. Consequently, the identical carrier frequencies in full-duplex system have no influence on applying the proposed analysis method and results to full-duplex system.

\section{Simulation Results and Analysis}

In this section, we will demonstrate the validity of the above analytical results through simulations operating in various scenarios, especially for expressions of (20), (26) and (27). The simulations are conditioned on varying adjustment factors without other biases, different attenuation biases with a fixed response-time and varying attenuator response-times without other biases.

All simulations in this section are operated as follows. Firstly, transmit and receive radios both adopt QPSK modulation with adjacent channel power ratio $-35 \mathrm{~dB}$, and the transmitting and receiving frequencies are respectively $54.5 \mathrm{MHz}$ and $54.75 \mathrm{MHz}$, and signal bandwidth is $50 \mathrm{kHz}$. On the other hand, the power of transmit signal is $37 \mathrm{dBm}$, and the desired signal is assumed to $-85 \mathrm{dBm}$. The reference signal power $P_{\mathrm{s}}$ is constrained to $10 \mathrm{dBm}$. The isolation between transmit and receive antennas is $35 \mathrm{~dB}$, that is, the receive self-interference power $P_{\mathrm{I}}$ is $2 \mathrm{dBm}$. Finally, the noise floor is $-110 \mathrm{dBm}$, and all the simulation results in this section are the average over 200 times. Consequently, the power spectrum of self-interference, desired signal and noise at the 


\begin{tabular}{|c|c|c|c|c|}
\hline $\begin{array}{c}\text { adjustment } \\
\text { factor } k\end{array}$ & $\begin{array}{c}\text { theory } \\
\mathrm{ICR}_{\infty}(\mathrm{dB})\end{array}$ & $\begin{array}{c}\text { simulation } \\
\mathrm{ICR}_{\infty}(\mathrm{dB})\end{array}$ & $\begin{array}{c}\text { theory } \\
\mathrm{SCT}_{\gamma=60}(\mathrm{~ms})\end{array}$ & $\begin{array}{c}\text { simulation } \\
\mathrm{SCT}_{\gamma=60}(\mathrm{~ms})\end{array}$ \\
\hline 10 & 96.76 & 96.81 & 0.984 & 1.033 \\
\hline 20 & 93.52 & 93.30 & 0.464 & 0.516 \\
\hline 40 & 90.00 & 89.39 & 0.203 & 0.248 \\
\hline
\end{tabular}

Tab. 1. Theory and simulation results of different adjustment factors.

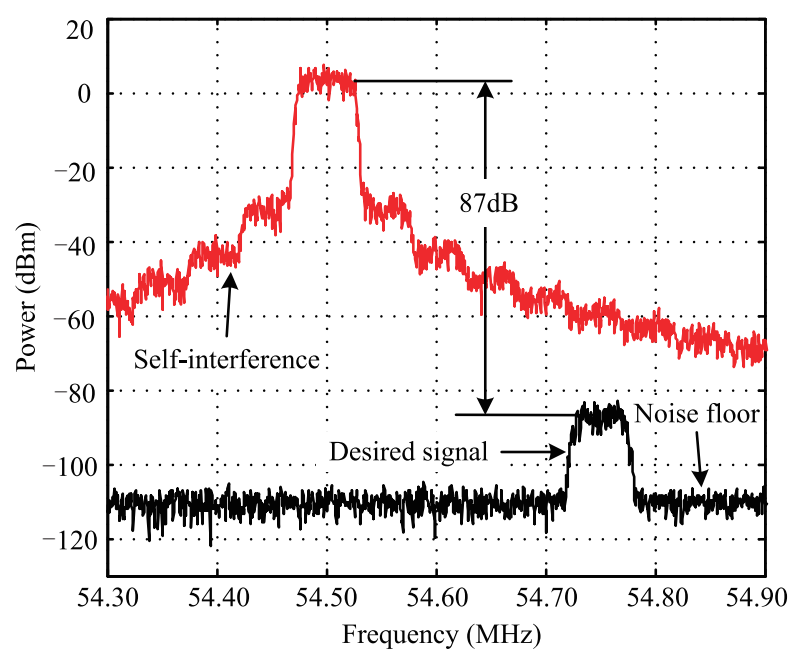

Fig. 3. Power spectrums of receive signals, including selfinterference, desired signal and noise.

receive radio antenna are shown in Fig. 3. Observing at the receive antenna, the self-interference power is stronger $87 \mathrm{~dB}$ than desired signal, and stronger $112 \mathrm{~dB}$ above the noise floor, and this is why RF adaptive interference cancellation should be applied in front of receive radio.

\subsection{Simulations for Varying Adjustment Fac- tors}

We can know that the stability $\mathrm{ICR}_{\infty}$ is mainly affected by adjustment factor $k$. Thus simulations are carried out for $k=10,20,40$ to analyze adjustment factor influence and to verify expressions (20) and (27). In this scenario, it is assumed that there are no delay bias and attenuation bias, and the execution interval time is $T_{\Delta}=15 \mu \mathrm{s}$ according to the response-time of actual attenuator [18]. To analyze the convergence speed, the SCT is defined as the time that ICR arriving at threshold $\gamma=60 \mathrm{~dB}$ consumes. With simulation parameters in this part, the theory values of $\mathrm{ICR}_{\infty}$ and SCT values can respectively be calculated through (20) and (27), and are listed in Tab. 1. Based on 200 times independent simulations, the cancellation results are shown as Fig. 4 and Tab. 1.

We can know from Fig. 4 that the adjustment factor would impact both $\mathrm{ICR}_{\infty}$ and SCT, and $\mathrm{ICR}_{\infty}$ would decrease more than $3 \mathrm{~dB}$ with adjustment factor doubled. It is well known that the above simulation results perfectly coincide with expression (20). Comparing simulation results with values of theoretical analysis in Tab. 1, it is found that they agree well, which demonstrates validity of ICR

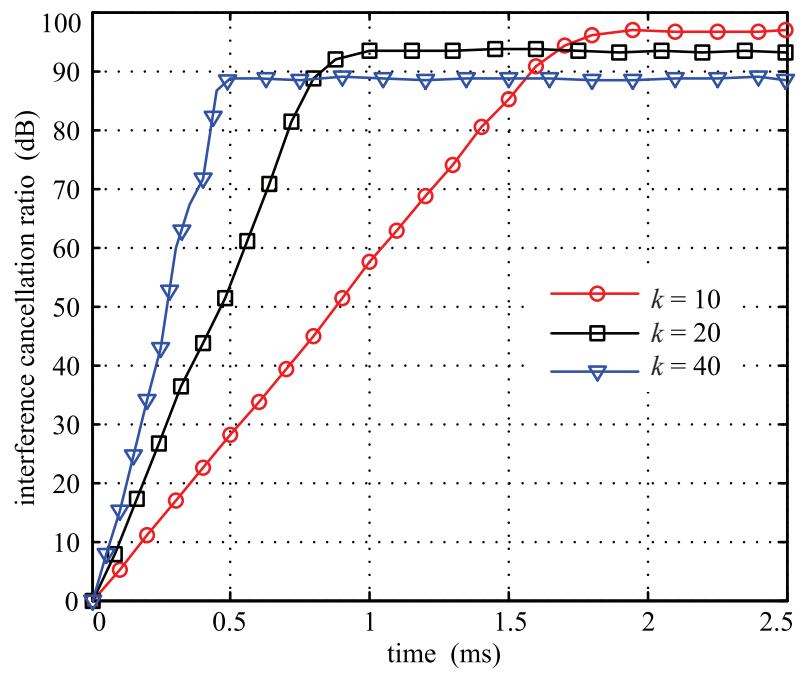

Fig. 4. ICR curves of simulations for different adjustment factors.

\begin{tabular}{|c|c|c|}
\hline $\begin{array}{c}\text { attenuation } \\
\text { bias } \sigma_{\varepsilon}\end{array}$ & $\begin{array}{c}\text { theory } \\
\mathrm{ICR}_{\infty}(\mathrm{dB})\end{array}$ & $\begin{array}{c}\text { simulation } \\
\mathrm{ICR}_{\infty}(\mathrm{dB})\end{array}$ \\
\hline $0.001 \sqrt{P_{\mathrm{I}}}$ & 72.52 & 72.49 \\
\hline $0.01 \sqrt{P_{\mathrm{I}}}$ & 52.55 & 52.42 \\
\hline $0.1 \sqrt{P_{\mathrm{I}}}$ & 32.55 & 32.46 \\
\hline
\end{tabular}

Tab. 2. Theory and simulation results of different attenuation biases.

expression (20) and SCT expression (27). With the above results and analysis, it draws a conclusion that, without delay and attenuation biases, the stability ICR can achieve more than $90 \mathrm{~dB}$ only if adjustment factor is not more than 20 . On the other hand, in Tab. 1, it is clear that the SCT is about $0.52 \mathrm{~ms}$ with adjustment factor equaling 20 , and the SCT reduces more than half with a double increase of adjustment factor. From above analysis, it can be concluded that ICR and SCT are both affected by adjustment factor; furthermore, the adjustment factor should be determined by the attenuator increment and response-time to achieve perfect performance of RFAICS.

\subsection{Simulations for Different Attenuation Biases}

In these simulations, we aim to analyze ICR degradation as a result of attenuation bias, thus the standard deviations $\sigma_{\varepsilon}$ of attenuation bias are set to three values $0.001 \sqrt{P_{\mathrm{I}}}, 0.01 \sqrt{P_{\mathrm{I}}}$ and $0.1 \sqrt{P_{\mathrm{I}}}$, where the $P_{\mathrm{I}}$ is $2 \mathrm{dBm}$. The adjustment factor and execution interval time are respectively assumed as 20 and $15 \mathrm{~ns}$. Simulation results and theory values of stability 


\begin{tabular}{|c|c|c|c|c|}
\hline $\begin{array}{c}\text { interval } \\
\text { time } T_{\Delta}(\mu \mathrm{s})\end{array}$ & $\begin{array}{c}\text { theory } \\
\mathrm{ICR}_{\infty}(\mathrm{dB})\end{array}$ & $\begin{array}{c}\text { simulation } \\
\mathrm{ICR}_{\infty}(\mathrm{dB})\end{array}$ & $\begin{array}{c}\text { theory } \\
\mathrm{SCT}_{\gamma=60}(\mathrm{~ms})\end{array}$ & $\begin{array}{c}\text { simulation } \\
\mathrm{SCT}_{\gamma=60}(\mathrm{~ms})\end{array}$ \\
\hline 10 & 96.76 & 96.81 & 0.984 & 1.033 \\
\hline 20 & 93.52 & 93.30 & 0.464 & 0.516 \\
\hline 40 & 90.00 & 89.39 & 0.203 & 0.248 \\
\hline
\end{tabular}

Tab. 3. Theory and Simulation results of different execution interval times.

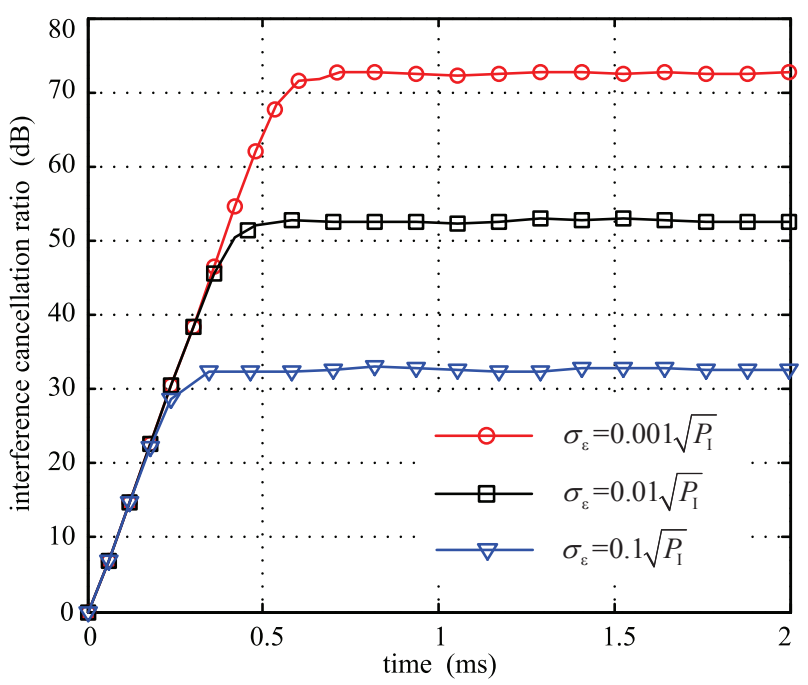

Fig. 5. ICR curves of different attenuation biases.

ICR calculated by (26) are listed in Tab. 2, and curves of three simulations are shown in Fig. 5.

Firstly, simulation results are also approximately equal to theory values with only about $0.1 \mathrm{~dB}$ difference. From Fig. 5, it is obvious that ICR is degraded seriously by the attenuation bias. On the other hand, compared with simulation results in Subsection A, standard deviation $0.01 \sqrt{P_{\mathrm{I}}}$ could deteriorate ICR to about $52 \mathrm{~dB}$, and the reduction is more than $40 \mathrm{~dB}$. Finally, theory analysis and simulation results both imply that the attenuation bias is the main barrier to improve ICR, thus we should apply attenuator with higher accuracy as far as possible in practical.

\subsection{Simulations for Varying Execution Interval Times}

Besides attenuation bias, response-time is another key parameter of attenuator since it determines the execution interval time. Therefore, simulations with different execution interval times are operated in this sub-section to analyze the effect of attenuator response-time on system convergence time. Similar to subsections A and B, with no attenuation bias, and fixed adjustment factor $k$, the interval time $T_{\Delta}$ values are respectively set to $12 \mu \mathrm{s}, 24 \mu \mathrm{s}$ and $36 \mu \mathrm{s}$. Simulation results and theory values for three conditions are shown in Tab. 3 and Fig. 6.

Considering (20) and (27), we can find that the execution interval time affects SCT rather than stability ICR, and this conclusion is verified by the simulation results in Tab. 3 . For the three assumed values of $T_{\Delta}, \mathrm{ICR}_{\infty}$ values are almost

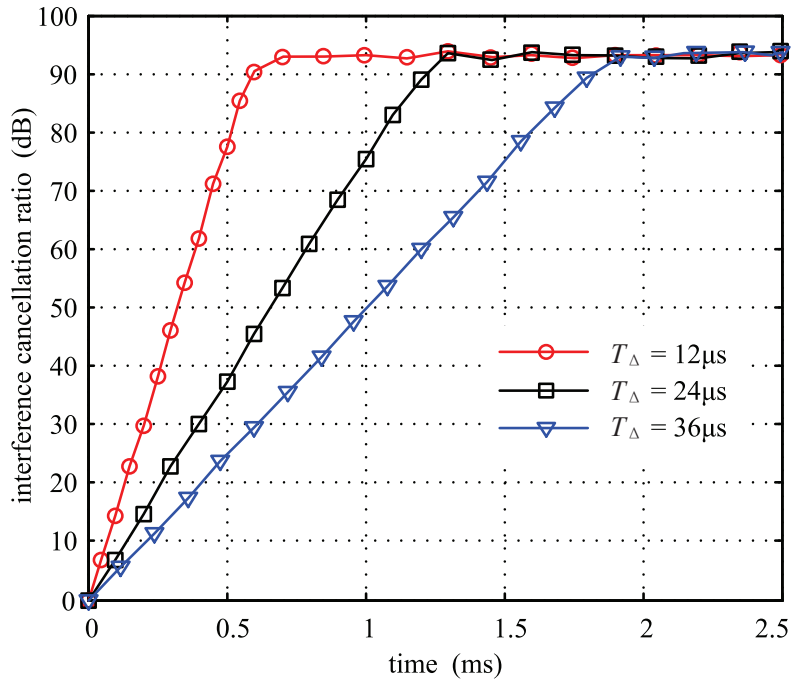

Fig. 6. ICR curves of different execution interval times.

unchanged; nevertheless their SCT are greatly different, and SCT increases linearly with the interval time $T_{\Delta}$. In addition, the simulation results and theory values listed in Tab. 3 also demonstrate the rationality of SCT expression.

In this section, simulations for different biases are executed to verify and analyze negative effects of imperfect attenuators on RFAICS performance. Simulation results show that if gain factor reduces by half, the stability ICR would increase more than $3 \mathrm{~dB}$, however, the SCT would disappointedly get doubled. Without analog device biases, the stability ICR can achieve more than $90 \mathrm{~dB}$ for $k=20$. On the other hand, the stability SICR reduces about $40 \mathrm{~dB}$ with attenuation bias $\sigma_{\varepsilon}=0.01 \sqrt{P_{\mathrm{I}}}$. Moreover, simulations for response-time indicate that the SCT would linearly increase with attenuator response-time and the SCT corresponding to $\mathrm{ICR}=60 \mathrm{~dB}$ is about $0.787 \mathrm{~ms}$ for interval time $T_{\Delta}=24 \mu \mathrm{s}$.

\section{Conclusion}

In this paper, the negative effects of non-ideal attenuators on RFAICS performance are analyzed in theory and verified in simulations. Firstly, based on the study of RFAICS convergence process, impact of attenuation bias on ICR is quantifiably investigated and analyzed, and we derive the comprehensive stability ICR expression. In addition to attenuation bias, how attenuator response-time affects SCT of RFAICS is also studied in detail, and the SCT expression is deduced for the first time. Finally, the simulations in varying scenarios demonstrate the validity of analytical results 
in this paper, which have significant guidance for practical applications. It is worth pointing out that research on other components, such as power amplifier and filter, should be executed in future, and the influence of the multipath reflection caused by different surround environments is also crucial for the suppression of electromagnetic interference between nearby radios working in the same platform.

\section{Acknowledgments}

This project is supported by the National Defense Research Foundation of China.

\section{References}

[1] KARAWAS, G., GOVERDHANAM, K., KOH J. Wideband active interference cancellation techniques for military applications. In Proceedings of the 5th European Conference on Antennas and Propagation (EUCAP 2011), Rome (Italy), 2011, p. 390-392. ISSN: 2164-3342

[2] SAUTER, W., GHOSE, R. Active Interference Cancellation Systems. 1st ed. Defense Technical Inforamtion Center, 1969.

[3] WISMER, L. D., ABRAMS, B. S. High Power Broadband Cancellation System. 1st ed. Defense Technical Information Center, 1981.

[4] LUSTGARTEN, M., HENSLER, T., MAIUZZO, M. A cosite analysis capability for evaluating the EMC of frequency hopping and single channel radios. In Proceedings of the Challenges of the 1990's Tactical Communications. Tactical Communications Conference. Fort Wayne (USA), 1990, p. 543-553. DOI: 10.1109/TCC.1990.177751

[5] MARTENS, S. M., MISCHI, M., OEI, S. G., et al. An improved adaptive power line interference canceller for electrocardiography. IEEE Transactions on Biomedical Engineering, 2006, vol. 53, no. 11, p. 2220-2231. DOI: 10.1109/TBME.2006.883631

[6] DEBAILliE, B., BROEK, D. J., LAVIN, C., et al. Analog/RF solutions enabling compact full-duplex radios. IEEE Journal on $\mathrm{Se}$ lected Areas in Communications, 2014, vol. 32, no. 9, p. 1662-1673. DOI: 10.1109/JSAC.2014.2330171

[7] ZHOU, J., CHUANG, T. H., DINC, T., et al. Integrated wideband self-interference cancellation in the RF domain for FDD and fullduplex wireless. IEEE Journal of Solid-State Circuits, 2015, vol. 50, no. 12, p. 3015-3031. DOI: 10.1109/JSSC.2015.2477043

[8] LIU, Y., QUAN, X., PAN, W., et al. Nonlinear distortion suppression for active analog self-interference cancellers in full duplex wireless communication. In Proceedings of the IEEE Globecom Workshops (GC Wkshps 2014). Austin (USA), 2014, p. 948-953. DOI: 10.1109/GLOCOMW.2014.7063555

[9] DUARTE, M., DICK, C., SABHARWAL, A. Experiment-driven characterization of full-duplex wireless systems. IEEE Transactions on Wireless Communications, 2012, vol. 11, no. 12, p. 4296-4307. DOI: 10.1109/TWC.2012.102612.111278

[10] KAUFMAN, B., LILLEBERG, J., AAZHANG, B. An analog baseband approach for designing full-duplex radios. In Proceedings of the Conference on Signals, Systems and Computers, Asilomar 2013. Pacific Grove (USA), 2013, p. 987-991. DOI: 10.1109/ACSSC.2013.6810438
[11] BHARADIA, D., MCMILIN, E., KATTI, S. Full duplex radios. In Proceedings of the Conference ACM SIGCOMM 2013. Hong Kong (China), 2013, p. 375-386. DOI: 10.1145/2486001.2486033

[12] WENLU, L., ZHIHUA, Z., JIAN, T., et al. Performance analysis and optimal design of the adaptive interference cancellation system. IEEE Transactions on Electromagnetic Compatibility, 2013, vol. 55, no. 6, p. 1068-1075. DOI: 10.1109/TEMC.2013.2265803

[13] WENLU, L., ZHIHUA, Z., YI, L., et al. Time-domain analysis of adaptive interference cancellation system with the desired signal. Journal of China Institute of Communications, 2012, vol. 33, no. 10, p. 183-190. DOI: 10.3969/j.issn.1000-436X.2012.10.024

[14] JOSEPH, G. M., KENNETH, E. K. Optimal tuning of analog self-interference cancellers for full-duplex wireless communication. In Proceedings of the 50th Annual Allerton Conference on Communication, Control, and Computing (Allerton), 2012. IIIinois (USA), 2012, p. 246-251. DOI: 10.1109/Allerton.2012.6483225

[15] HUUSARI, T., CHOI, Y. S., LIIKKANEN, P., et al. Wideband selfadaptive RF cancellation circuit for full-duplex radio: operating principle and measurements. In Proceedings of the 81st IEEE Vehicular Technology Conference (VTC Spring). Glasgow (UK), 2015, p. 1-7. DOI: 10.1109/VTCSpring.2015.7146163

[16] HAYKIN, S. S. Adaptive Filter Theory. 4th ed. Pearson Education, 2008. ISBN: 9788131708699

[17] DOGAN, H., MEYER, R. G., NIKNEJAD, A. M. Analysis and design of RF CMOS attenuators. IEEE Journal of Solid-State Circuits, 2008, vol. 43, no. 10, p. 2269-2283. DOI: 10.1109/JSSC.2008.2004325

[18] PEREGRINE SEMICONDUCTOR. A HaRP $P^{\mathrm{TM}}$-enhanced, high linearity, 6-bit RF digital step attenuator (DSA) (PE43601). Product Specification. 13 pages. [Online] Cited 2016-03-11. Available at: http://www.psemi.com/pdf/datasheets/pe43601ds.pdf

\section{About the Authors}

Jian-cheng LIU (corresponding author) was born in Handan, China in July 1987. He received his B.E. degree and M.E. degree from Electronic Engineering Institute of PLA respectively in 2010 and 2013. His research interests include anti-jamming technology of wireless communication and adaptive communication signal processing.

Hou-de QUAN was born in Dalian, China in 1963. He received his PhD. degree from Northwestern Polytechnical University, China in 2008. Now he is a professor in Dept. of Information Engineering of Mechanical Engineering College. His research interests include information and communication engineering.

Pei-zhang CUI was born in Changzhi, China in 1974. He received his M.E. degree from the 54th Research Institute of CETC, China in 2005.

Hui-xian SUN was born in Baotou, China in 1980. He received his M.E. degree and $\mathrm{PhD}$. degree from National University of Defense Technology respectively in 2005 and 2010. 